\title{
Intracranial dissemination of pituitary adenomas
}

\author{
KATHLEEN M. OGILVY AND JAN JAKUBOWSKI \\ From the Departments of Neuropathology and Neurological Surgery, \\ The Royal Infirmary, Sheffield
}

SUMmaRY Two unusual cases of pituitary adenomas giving distant secondary deposits inside the cranial cavity are presented. The authors comment on the accessible literature and the possible routes and cause of the spread. Finally they discuss the problematic nomenclature and classification of these tumours.

The great majority of pituitary tumours are entirely benign and circumscribed. There is, however, a small but important group of these tumours which behave in a more aggressive manner, giving rise to infiltration of related structures, or-more rarely-remote metastases.

Well-documented cases of metastasizing pituitary adenomas are rare. Willis (1934) examined the few recorded cases up to that date and concluded that they were fallacious. Since then, extracranial metastases have been reported by Cohen and Dible (1936), Forbes (1947), Sheldon, Golden, and Bondy (1954), Salassa, Kearns, Kernohan, Sprague, and MacCarty (1959), Scholz, Gastineau, and Harrison (1962) and Geroulanos (1969). The majority of these authors (Cohen and Dible, Forbes, Sheldon, Geroulanos) accepted the biological malignancy of these tumours, irrespective of the histological appearance, and called them carcinomas by definition. Scholz et al. (1962) commented on the interesting fact that these cases were often associated with Cushing's syndrome.

Locally invasive adenomas which infiltrate contiguous structure, notably the cavernous sinuses, are a well-recognized, if uncommon, clinicopathological entity. Jefferson (1955) described 14 such cases in his Sherrington lecture. All showed histological evidence of aggressive growth, with nuclear pleomorphism and mitotic activity, and in three cases there was actual invasion of either nerve bundles or the carotid adventitia. In 1965 Martins, Hayes, and Kempe described five cases of invasive pituitary adenoma collected over a period of 11 years. In both these series, spread of the tumour appeared to be by direct extension, either via the meninges or along nerve bundles. A possible exception could be case $\mathrm{V}$ in Jefferson's series, in which multiple, apparently isolated tumour nodules were found in the dura mater of the middle fossa. Solitare and Jatlow (1967) described an interesting case of an invasive pituitary tumour which not only infiltrated related structures but produced three isolated deposits on the ventral aspect of the right frontal lobe, the largest being $5 \mathrm{~mm}$ in diameter. These deposits were infiltrating brain tissue for distances of up to $5 \mathrm{~mm}$. In view of the rarity of distant intracranial secondary deposits from pituitary tumours, we would like to present and discuss the following two unusual cases.

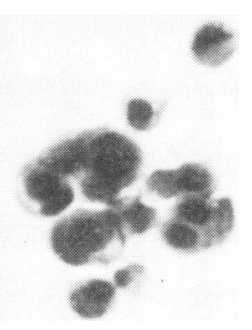

FIG. 1. Case 1. Centrifuged deposit from lumbar CSF, showing clump of tumour cells. $H$ and $E, \times 250$. 
CASE 1

This 54 year old man was admitted to the Neurosurgical Department in March 1967, with a few months' history of progressive visual deterioration,
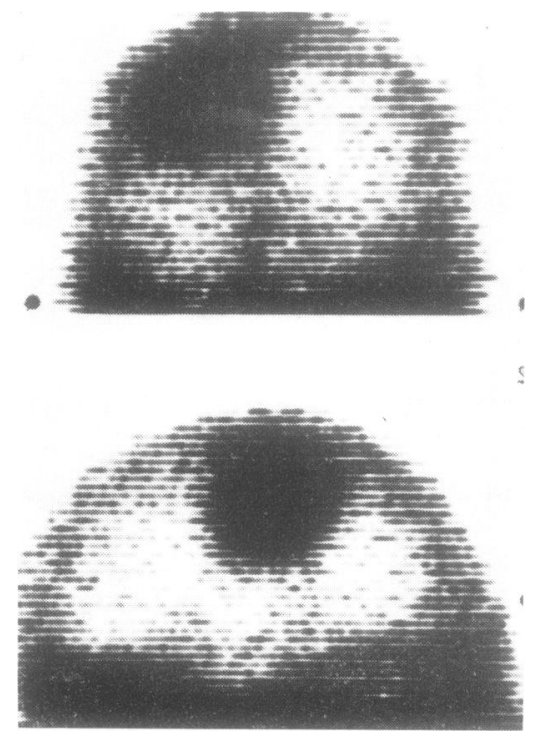

FIG. 2. Case 1. Gamma-scan showing area of very high uptake in the left parietal parasagittal region. The appearance is characteristic of a benign, slowgrowing tumour. headache, and vomiting. On examination, there wases severely diminished visual acuity, particularly in the left eye, complete loss of the left nasal field, and some 0 diminution of the right temporal field. Otherwise physical examination was normal, and biochemical investigations of blood and urine were within normal limits. There was radiological evidence of a pituitary. fossa tumour with suprasellar extension.

A right frontal craniotomy was carried out and $a_{\frac{\rho}{0}}$ soft tumour which expanded above the sella turcica $\frac{-}{\circ}$ compressing the optic nerves was removed intra- $-\overline{\bar{c}}$. capsularly by the subfrontal route. Histologically, $\underset{\mathbb{D}}{ }$ this was a typical benign chromophobe adenoma. After operation the patient made a very satisfactory recovery. He then received a course of $x$-ray therapy, $\overrightarrow{0}$ and maintenance doses of cortisone $(12.5 \mathrm{mg}$ b.d.) $\overrightarrow{\vec{H}}$ and thyroxine $(0.1 \mathrm{mg}$ daily) were prescribed.

He remained well until August 1971, when he was readmitted because of gradually developing right hemiparesis, confusion, and nominal aphasia. Hise visual acuity and visual fields were at the same levelis as previously noted. A pneumoencephalogram $\overrightarrow{0}$ showed no evidence of recurrence of the pituitarye fossa tumour, but a sample of cerebrospinal fluid음 (CSF) removed during this investigation containedgroups of tumour cells (Fig. 1). A left carotid angie $-\frac{D}{O}$ gram suggested the possibility of a parietal, pa通sagittal mass and a gamma scan clearly demo $-\overrightarrow{0}$ strated a rounded area of very high uptake of isotope in the suspected region (Fig. 2).

A left temporoparietal craniotomy was performed@ and the tumour was found lying on the surface of the brain in the parietal parasagittal region. It was

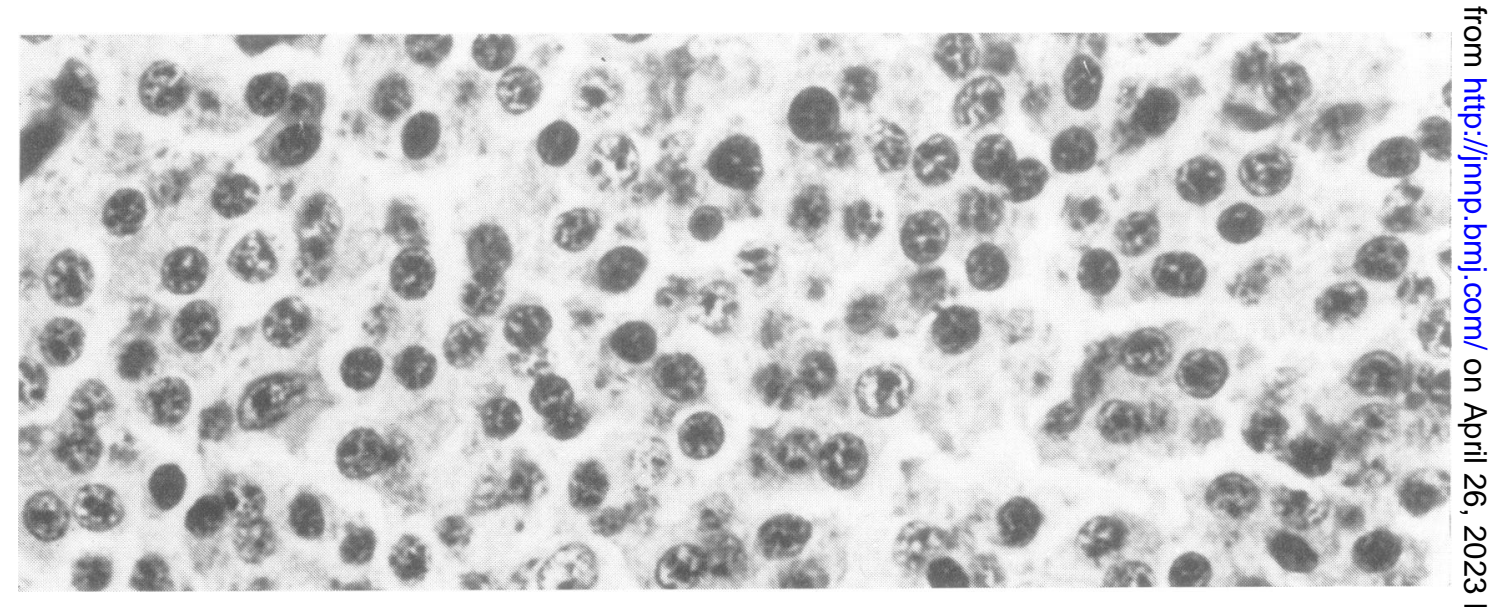

FIG. 3. Case 1. Parasagittal tumour deposit. Diffuse type of chromophobe adenoma. Compact arrangement of ill-defined polygonal cells. Some nuclear pleomorphism with occasional giant cell types and scattered mitoses. Some show atypical clumping of nuclear chromatin. $H$ and $E, \times 400$. 


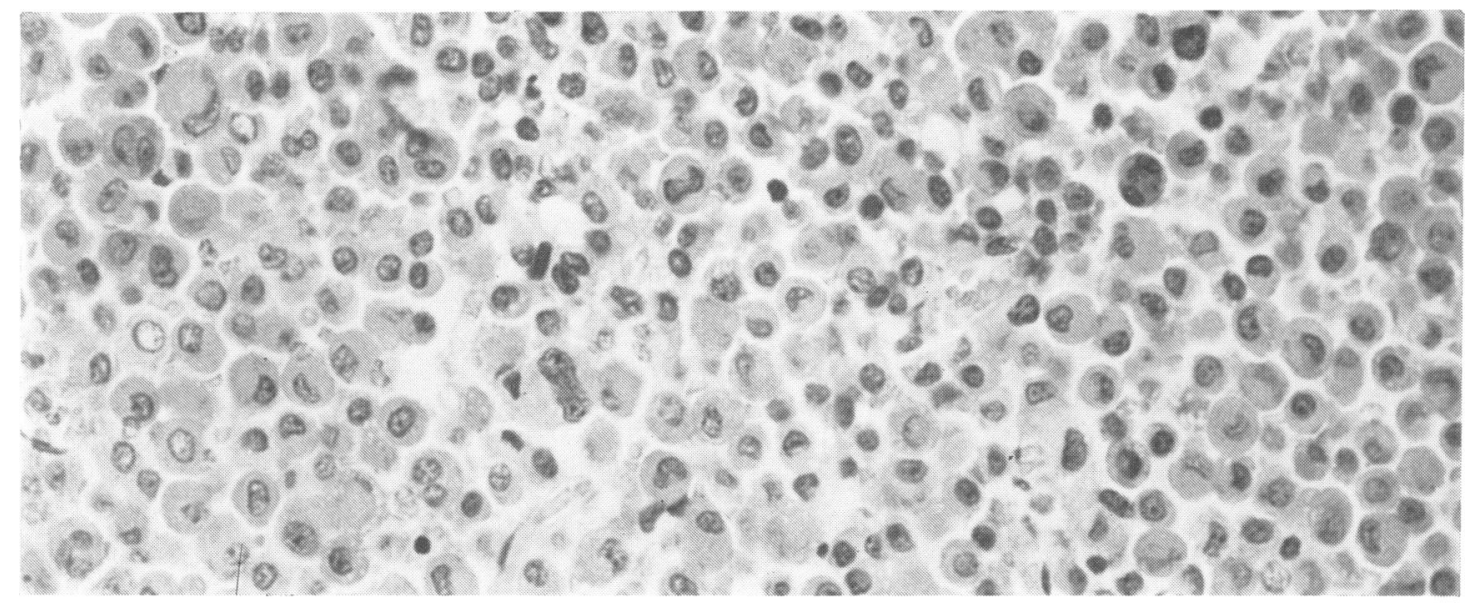

(a)

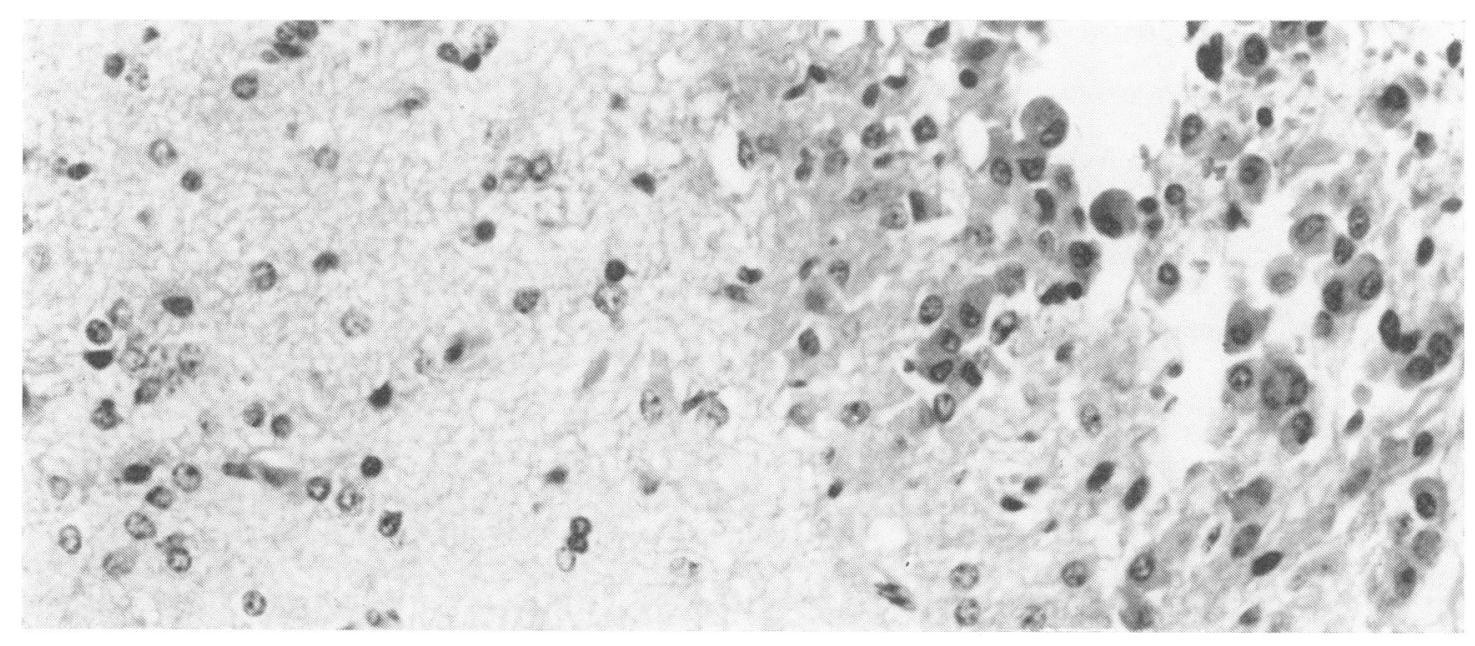

(b)

FIG. 4. Case 2. (a) Original pituitary adenoma, showing diffuse arrangement of well-defined polygonal cells containing acidophilic cytoplasmic granules (demonstrable by PAS/Orange G staining method). Nuclear pleomorphism with binucleate and multinucleate giant cells. Nuclei mainly vesicular, but some show atypical clumping of chromatin. $H$ and $E, \times 400$. (b) Superficial infiltration of olfactory tract by secondary deposit from acidophil adenoma. $H$ and $E, \times 400$.

attached to the inner aspect of the parietal dura mater and to the falx, and was surrounded by large cortical veins. The sagittal sinus was not invaded. A total excision of the mass, together with attached dura mater was achieved fairly easily as the tumour was circumscribed and was not infiltrating brain tissue. At operation it was thought to be a meningioma. After removal, the tumour was found to weigh $22 \mathrm{~g}$ and it measured $4.5 \times 5.0 \times 1.5 \mathrm{~cm}$. The microscopic appearance came as a complete sur- 


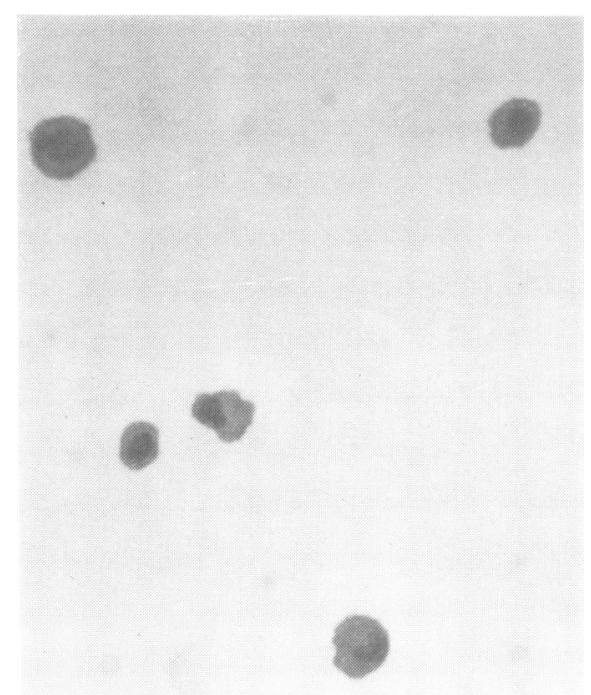

FIG. 5. Case 2. Centrifuged deposit from lumbar CSF, showing scattered tumour cells. $H$ and $E, \times 400$.

prise, as it was found to be a chromophobe adenoma. Although non-invasive it now appeared more actively growing, and pleomorphic (Fig. 3).

Postoperatively, the patient made a complete recovery and was discharged five weeks later for a further course of radiotherapy.

\section{CASE 2}

This 49 year old female patient was admitted to the Neurosurgical Department in October 1968, having been a known acromegalic for nine years, and having received a course of radiotherapy in 1964 . She was admitted because of deteriorating vision and violent headaches.

On examination she was a typical acromegalic. Visual acuity was severely reduced, particularly in the left eye, and there was a definite left temporal field defect. Biochemical examinations of the blood and urine were normal. Radiographs of the skull showed that the pituitary fossa was enlarged and eroded and that there was pronounced frontal hyperostosis. Pneumoencephalography showed a $1.5 \mathrm{~cm}$ suprasellar extension indenting the anterior wall and floor of the third ventricle.

A right frontal craniotomy was carried out. On retracting the right frontal lobe, an isolated mass measuring approximately $1 \mathrm{~cm} \times 7 \mathrm{~mm} \times 4 \mathrm{~mm}$ was found adherent to the olfactory tract and this was excised. The main tumour mass was suprasellar in position, lying between the two optic nerves, and appeared unencapsulated. As much as was possible was removed piecemeal.

Histological examination of the suprasellar mass showed a predominantly acidophilic type of adenoma, containing groups of chromophobe cells. Although pleomorphic, with many giant cell types, there was no evidence of mitotic activity (Fig. 4a). It was interesting however to note that sections of the mass adherent to the olfactory tract showed superficial infiltration of the tract by similar tumour (Fig. $4 \mathrm{~b}$ ). At the time of operation it was supposed that this deposit had arisen as a result of seeding from the unencapsulated tumour mass along the arachnoidal 6 sleeve surrounding the olfactory tract. After the $\vec{\circ}$ operation, she made a good recovery, and visual acuity in the left eye improved. She was discharged three weeks later on maintenance doses of cortisone $12.5 \mathrm{mg}$ b.d. and thyroxine $0.1 \mathrm{mg}$ daily.

She remained well for five months, then came back to the outpatient department complaining of nausea and vomiting of three weeks' duration, visual deterioration, and pain in the distribution of the first division of the right trigeminal nerve. In view of the operative findings, it seemed obvious that she had $\&$ further spread of the tumour. Confirmatory evE $D$ dence of dissemination was obtained when the lumbar CSF was found to contain cells similar to those forming the original tumour (Fig. 5). She wh referred to the radiotherapists for irradiation of the whole head. After this her condition fluctuated until her death in January 1971.

Examination of the cranial cavity at necropsy showed soft tumour in the pituitary fossa directly extending into both cavernous sinuses, and also through the eroded floor of the pituitary fossa into the sphenoidal air sinuses and nasopharynx. On the right side, tumour had extended from the cavernous $\supset$ sinus to involve the trigeminal ganglion and from there had spread along the trigeminal roots producing a mass $5 \mathrm{~cm}$ in diameter in the cerebellopontine angle, which had displaced the brain-stem to the left. The left trigeminal ganglion and nerve were also expanded 3 . by tumour. Several nodules of tumour all less than 8 $1 \mathrm{~cm}$ in diameter were found in the dura mater of the middle and posterior cranial fossae on both sides. At 0 first sight, these nodules appeared discrete, but on stripping the dura mater from the bone, the outer aspect was seen to be covered by a sheet of yellowish granular tumour which was in direct continuity with $N$ the infiltrated cavernous sinuses. Two further dis- $N$ crete deposits were found in the subarachnoid space N overlying both temporal poles. On the right side, the $\sigma \sigma$ deposit measured $1.5 \mathrm{~cm}$ in diameter. The corresponding measurement on the left was $1.2 \mathrm{~cm}$. These deposits appeared quite separate from the main $\stackrel{\oplus}{+}$ 


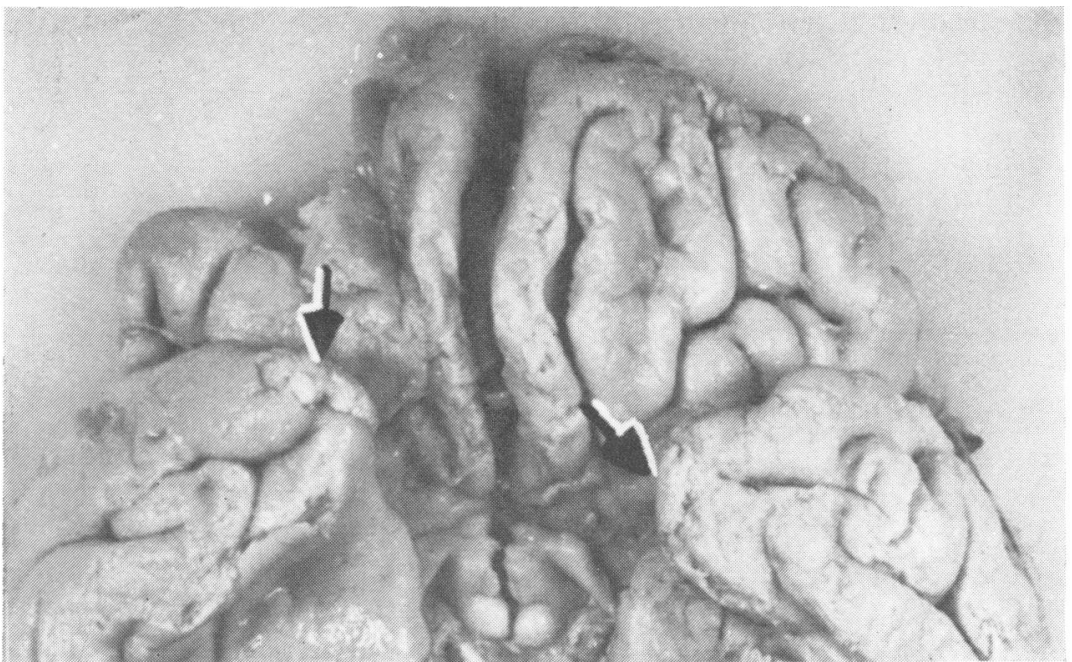

(a)

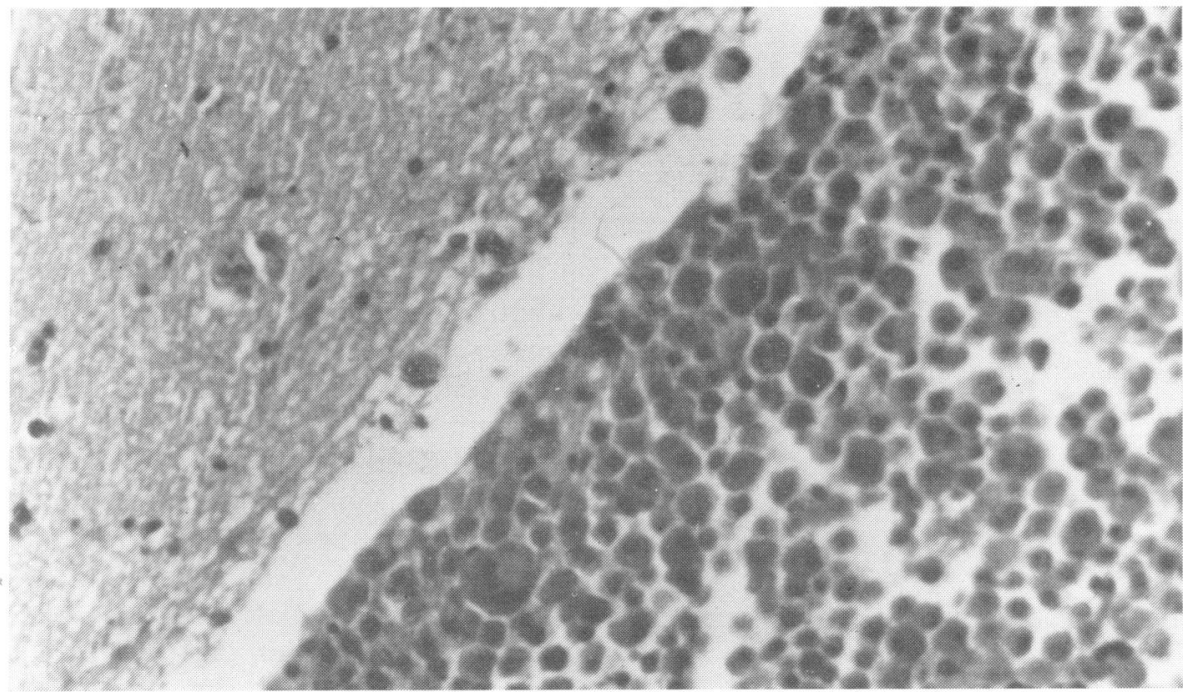

(b)

FIG. 6. Case 2. (a) Ventral aspect of brain showing tumour deposits (indicated by arrows) in subarachnoid space overlying both temporal poles. (b) A section of one of these deposits showing the subarachnoid position of the tumour. A few tumour cells are just within the molecular layer of the underlying temporal lobe cortex. $H$ and $E, \times 400$. 
tumour mass, and were confined to the subarachnoid space (Figs 6a and 6b).

Histological examination of the tumour showed that it had altered slightly in character during the 14 months which had elapsed since operation, and had become more pleomorphic and anaplastic. Sections of infiltrated trigeminal nerve showed actual destruction and replacement of nerve bundles (Fig. 7).

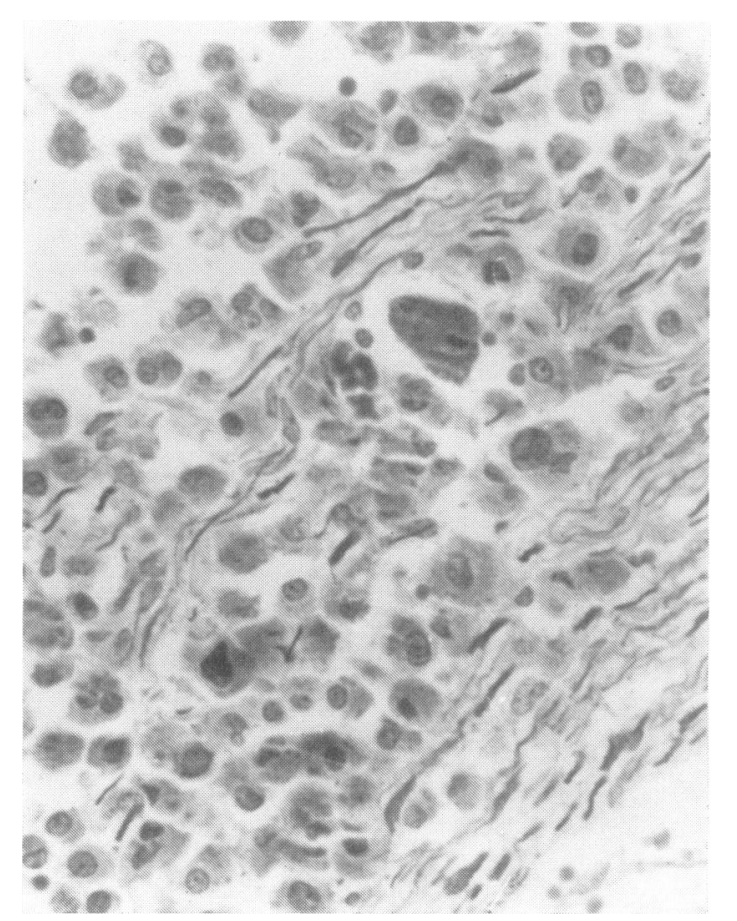

FIG. 7. Case 2. Portion of trigeminal nerve root to show actual invasion and destruction of nerve fibres by tumour. Holmes's silver, $\times 400$.

\section{DISCUSSION}

Dissemination of such tumours as ependymomas and medulloblastomas within the subarachnoid space is familiar. The frequency of this dissemination is presumably linked to their intimate relationship with the ventricular system. However, seedling deposits from these tumours are almost entirely confined to the basal and spinal regions.

The possible methods of intracranial dissemination of pituitary tumours requires analy- sis. Apart from infiltration of contiguous structures, spread may, theoretically, occur via the blood stream, the meninges, or the cerebrospinal fluid circulation. Each of these methods was considered with reference to the foregoing two cases.

The hypophysis is surrounded by a rich plexus of venous channels, and pituitary adenomas have the ability to infiltrate the walls of veins without having become truly malignant. A non-occlusive infiltration of these venous channels could give rise to retrograde venous embolism via the great anastomotic vein of Trolard, particularly as the cerebral veins are devoid of valves. Although the investigation of these two cases gives no specific support for this theory, the position of the lesion in case 1 is such that the possibility of blood-borne metastasis cannot be excluded entirely.

Under normal conditions there is no point of entry for cerebrospinal fluid into the subdural space. However, during operation on a pituitary adenoma, the subarachnoid space, and in paro ticular the chiasmatic cistern, is inevitably opened and cerebrospinal fluid leaks into the subdural space. It might therefore be possible $\overrightarrow{0}$

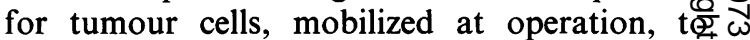
escape by this route. In addition any tumour which has penetrated the arachnoid would, theoretically, be in a position to disseminate itself subdurally. However, in neither of our cases is there any positive evidence of this kind of subdural spread. We wish to stress that the subdural nodules encountered in case 2 have penetrated the dura mater from an extradural sheet of tumour which was in continuity with the main mass.

Although there is no extension of the subarachnoid space within the hypophyseal fossa, a pituitary tumour infiltrating the diaphragma sellae could readily shed clumps of cells into the interpeduncular, chiasmatic, or carotid cisterns Alternatively, tumour particles could enter the subarachnoid space during operation. Provided the particles are then able to negotiate the arachnoidal network, they may finally become arrested in an arachnoidal granulation, and here find favourable conditions for further growth We have concluded that the parasagittal deposit in case 1 most probably resulted from spread by the cerebrospinal fluid circulation. 
There appear to have been three modes of spread in case 2. Firstly, there is evidence of extradural spread of tumour with subsequent penetration of the dura mater. Secondly, there is evidence of direct extension along nerve bundles, producing the mass in the cerebellopontine angle. Finally it seems overwhelmingly probable that subarachnoid spread by the CSF circulation accounted for the olfactory tract deposit observed at operation, and the temporal pole deposits found at necropsy.

In both cases the presence of tumour cells in the lumbar cerebrospinal fluid was detected during life. This is an interesting observation for two reasons. On the one hand, it strongly supports the theory of transmission of tumour particles via the CSF circulation, and, on the other hand, we feel that this may be an important diagnostic feature in cases of pituitary tumour with intracranial or intraspinal dissemination. With this in mind, we reviewed all pituitary adenomas treated by operation in the Sheffield Department of Neurosurgery over the past 20 years. Out of a total number of 97 , the lumbar CSF deposit was examined in 20 cases. Tumour cells were detected in only two of these-namely, the cases reported here-and in none of the remaining 18 was there clinical or operative evidence of dissemination of the tumour.

Case 1 underlines the fact that the histological appearance of a pituitary adenoma may bear little relationship to its biological behaviour. The tumour was not malignant histologically, and yet it produced a discrete and remote secondary growth. Metastasis is defined by Willis (1967) as 'the transfer of detached tumour particles within vascular, coelomic, cerebrospinal or epithelial channels, and their successful lodgement and survival to produce discrete secondary growths at new sites'. By this definition, case 1 should be regarded as a metastasizing pituitary adenoma, although we would prefer the term 'seedling deposit'.

In case 2 , the tumour was histologically much more malignant and at operation showed the highly unusual features of penetration of the capsule and dissemination. The additional widespread dissemination encountered at necropsy seemed to indicate a high level of biological malignancy. It may be argued that the classical criterion of malignancy-that is, extracranial metastasis-is absent. This objection becomes insignificant when one recalls that glial tumours which are manifestly of a malignant nature almost invariably fail to produce extracranial secondary deposits. It seems likely that pituitary tumours share with glial tumours the almost total inability to survive outside the familiar and favourable nutritive environment of the cranial cavity.

However, the pedantic classification of these two tumours into either a benign or malignant category has little clinical relevance to the subarachnoid spread of pituitary tumours such as we have described.

It is a pleasure to record our gratitude to Dr. $\mathrm{H}$. Lederer of Doncaster Royal Infirmary for performing the necropsy in case 2 , and to $\mathrm{Mr}$. Antony Jefferson and Mr. James Hardman for valuable criticism and help in preparing this paper.

\section{REFERENCES}

Cohen, H., and Dible, J. H. (1936). Pituitary basophilism associated with basophil carcinoma of the anterior lobe of the pituitary gland. Brain, 59, 395-407.

Forbes, W. (1947). Carcinoma of the pituitary gland with metastases to the liver in a case of Cushing's syndrome. Journal of Pathology and Bacteriology, 59, 137-144.

Geroulanos, S. (1969). Chromophobes Hypophysenkarzinom mit Leber- und Knochenmetastasen. Schweizerische Medizinische Wochenschrift, 99, 1817-1824.

Jefferson, Sir G. (1955). The Invasive Adenomas of the Anterior Pituitary. Sherrington Lectures No. 3. University Press: Liverpool.

Martins, A. N., Hayes, G. J., and Kempe, L. G. (1965). Invasive pituitary adenomas. Journal of Neurosurgery, 22, 268-276.

Salassa, R. M., Kearns, T. P., Kernohan, J. W., Sprague, R. G., and MacCarty, C. S. (1959). Pituitary tumors in patients with Cushing's syndrome. Journal of Clinical Endocrinology, 19, 1523-1539.

Scholz, D. A., Gastineau, C. F., and Harrison, E. G., Jr. (1962). Cushing's syndrome with malignant chromophobe tumor of the pituitary and extracranial metastasis. Report of a case. Proceedings of the Staff Mectings of the Mayo Clinic, 37, 31-42.

Sheldon, W. H., Golden, A., and Bondy, P. K. (1954). Cushing's syndrome produced by pituitary basophil carcinoma with hepatic metastases. American Journal of Medicine, 17, 134-142.

Solitare, G. B., and Jatlow, P. (1967). Adenohypophysial carcinoma. Case report. Journal of Neurosurgery, 26, 624632.

Willis, R. A. (1934). The Spread of Tumours in the Human Body, p. 138. Churchill: London.

Willis, R. A. (1967). Pathology of Tumours, 4th edn. Butterworths: London. 